\title{
Evaluation of an imputed pitch velocity model of the auditory tau effect
}

\author{
Molly J. Henry \\ Bowling Green State University, Bowling Green, Ohio \\ J. DeVIN MCAuley \\ Michigan State University, East Lansing, Michigan \\ AND \\ Marta Zaleha \\ Bowling Green State University, Bowling Green, Ohio
}

\begin{abstract}
This article extends an imputed pitch velocity model of the auditory kappa effect proposed by Henry and McAuley (2009a) to the auditory tau effect. Two experiments were conducted using an AXB design in which listeners judged the relative pitch of a middle target tone $(\mathrm{X})$ in ascending and descending three-tone sequences. In Experiment 1, sequences were isochronous, establishing constant fast, medium, and slow velocity conditions. No systematic distortions in perceived target pitch were observed, and thresholds were similar across velocity conditions. Experiment 2 introduced to-be-ignored variations in target timing. Variations in target timing that deviated from constant velocity conditions introduced systematic distortions in perceived target pitch, indicative of a robust auditory tau effect. Consistent with an auditory motion hypothesis, the magnitude of the tau effect was larger at faster velocities. In addition, the tau effect was generally stronger for descending sequences than for ascending sequences. Combined with previous work on the auditory kappa effect, the imputed velocity model and associated auditory motion hypothesis provide a unified quantitative account of both auditory tau and kappa effects. In broader terms, these findings add support to the view that pitch and time relations in auditory patterns are fundamentally interdependent.
\end{abstract}

Lawful movement trajectories of objects in the world allow a perceiver to anticipate where an object will be when (Gibson, 1966; Shepard, 1984). Deviation of objects from their expected trajectory often results in errors in perception (Abbe, 1936; Abe, 1935; Benussi, 1913; Cohen, Hansel, \& Sylvester, 1953, 1954; Geldreich, 1934). The kappa effect refers to errors in perceived stimulus timing that are attributable to deviations from expected stimulus spacing (Cohen, Hansel, \& Sylvester, 1953, 1955; Huang \& B. Jones, 1982; B. Jones \& Huang, 1982; MacKenzie, 2007; Matsuda \& Matsuda, 1979, 1981; Price-Williams, 1954; Sarrazin, Giraudo, Pailhous, \& Bootsma, 2004); stimuli that are unexpectedly close together in space tend to be perceived as close together in time. Conversely, the tau effect refers to errors in perceived stimulus spacing that are attributable to deviations from expected stimulus timing (Benussi, 1913; Bill \& Teft, 1969; Christensen \& Huang, 1979; Cohen, Christensen, \& Ono, 1974; Cohen et al., 1954; Geldreich, 1934; Helson \& King, 1931; Huang \& B. Jones, 1982; B. Jones \& Huang, 1982); stimuli that are unexpectedly close together in time tend to be perceived as close together in space.
The canonical task used to investigate the visual kappa and tau effects involves an AXB design; the spatial and temporal positions of the first and third (bounding) stimuli (A and B) of a series of three stimuli are fixed, whereas the spacing and timing of the middle target stimulus (X) vary relative to the spatial and temporal bisection points of the bounding stimuli (see Figure 1). Thus, the threeelement sequence marks out two spatial intervals ( 1 1, s2) and two temporal intervals ( $\mathrm{t} 1, \mathrm{t} 2)$, with the spatial and temporal bisection points corresponding to $\mathrm{s} 1=\mathrm{s} 2$ and $\mathrm{t} 1=\mathrm{t} 2$, respectively.

In kappa studies in which the effects of stimulus spacing on perceived stimulus timing are considered, observers typically judge whether the middle target element is closer in time to either the first (A) or the third (B) bounding element by making a binary short-long versus longshort decision about the temporal pattern of the threeelement sequence or by explicitly adjusting the timing of the target element so that it subjectively bisects the time interval marked by the bounding elements. Under these conditions, observers tend to make systematic errors such that when $\mathrm{s} 1<\mathrm{s} 2$, the pattern of durations is perceived as short-long ( $\mathrm{t} 1<\mathrm{t} 2)$, even when the pattern of dura-

J. D. McAuley, dmcauley@msu.edu 

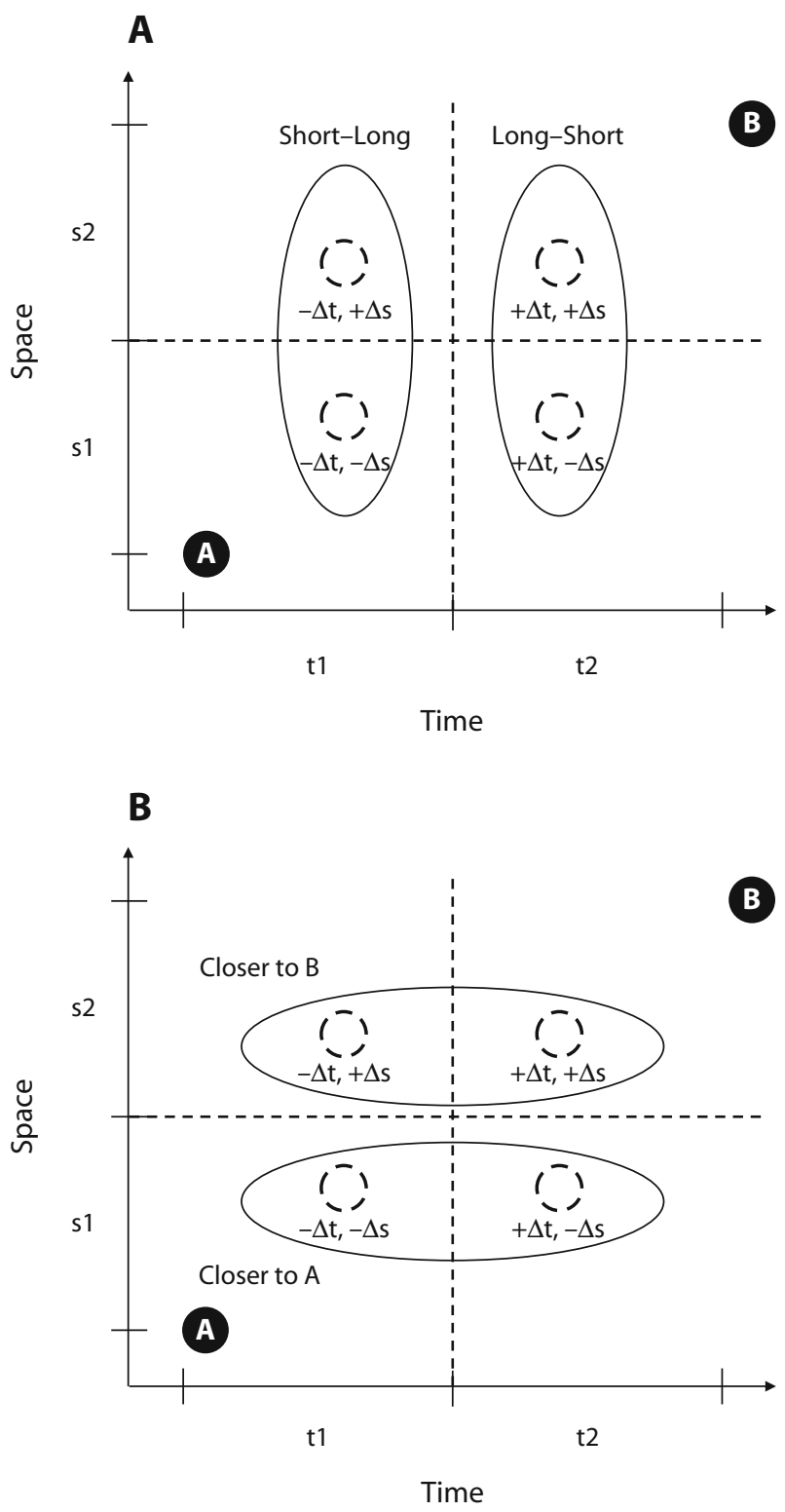

Figure 1. The canonical kappa and tau tasks. Participants experience three-element sequences; the first element (A) and the third element (B), indicated by closed circles, have fixed positions in space and time, whereas the spatial and temporal positions of the target element ( $X$; open circles) are varied from trial to trial, relative to the spatial and temporal bisection points of the initial and final (bounding) elements $(\mathrm{s} 1=\mathrm{s} 2$ and $\mathrm{t} 1=\mathrm{t} 2$, respectively; dotted lines). (A) For the canonical kappa task, participants judge the patterning of the two temporal intervals delineated by each three-element sequence, responding short-long or long-short. (B) For the canonical tau task, participants judge the patterning of the two spatial intervals delineated by each three-element sequence, responding closer to $A$ or closer to $B$.

tions is objectively long-short $(\mathrm{t} 1>\mathrm{t} 2)$. Similarly, when $\mathrm{s} 1>\mathrm{s} 2$, observers tend to perceive the pattern of durations as long-short (t1 > t2) (Abbe, 1936; Abe, 1935; Cohen et al., 1953).

Conversely, in tau studies in which the effects of stimulus timing on perceived stimulus spacing are considered, observers typically judge whether the middle target element is closer in space to either the first or the third bounding element by making a binary closer to $A$ versus closer to $B$ decision or by explicitly adjusting the spacing of the target element so that it subjectively bisects the distance marked by the bounding elements. Under these conditions, observers tend to make systematic errors such that, when $\mathrm{t} 1<\mathrm{t} 2$, the target is perceived as closer to $A$ $(\mathrm{s} 1<\mathrm{s} 2)$, even when the target is objectively closer to $B$ $(\mathrm{s} 1>\mathrm{s} 2)$. Similarly, when $\mathrm{t} 1>\mathrm{t} 2$, observers tend to perceive that the target is closer to $B(\mathrm{~s} 1>\mathrm{s} 2)$ (Benussi, 1913; Bill \& Teft, 1969, 1972; Geldreich, 1934; Huang \& B. Jones, 1982; B. Jones \& Huang, 1982).

In the present article, we consider an auditory version of the tau effect involving tone sequences and variations in frequency spacing of the tones, rather than their separation in physical space. This work parallels a recent study on the auditory kappa effect (Henry \& McAuley, 2009a). The motivation for conceptualizing frequency and the perceptual correlate, pitch, as a spatial dimension comes from a number of sources (Crowder \& Neath, 1995; Douglas \& Bilkey, 2007; Handel, 1988; Kubovy, 1981). First, in music, notes are typically thought of as having a pitch that varies in tone height; that is, musical notes can be high or low. This is visually apparent by the way that many cultures represent musical notes on a stave. Second, there is an analogy at the neural level between the topographic organization of the response of the retina to light and the tonotopic organization of the response of the basilar membrane to sound (Crowder \& Neath, 1995). Third, there is evidence that mental representations for musical pitch overlap to some degree with mental representations of physical space (Douglas \& Bilkey, 2007; Rusconi, Kwan, Giordano, Umiltà, \& Butterworth, 2006).

The remainder of the introduction consists of a review of research on the visual and auditory kappa and tau effects, followed by an overview of the present study.

\section{Visual Kappa and Tau Effects: Theory and Data}

The dominant explanation for the kappa and tau effects is based on an imputed velocity hypothesis (Collyer, 1977; B. Jones \& Huang, 1982). This hypothesis proposes that properties of moving objects are applied by the perceiver to displays made up of successive transient sequence elements, even though the displays are not explicitly moving per se. Because perceivers have a wealth of experience with moving objects in the environment that show coherent transformations in space and time constrained by physical laws, they tend to naturally apply these laws to artificial stimulus displays (Shepard, 1984). In the context of the canonical kappa and tau tasks, observers are hypothesized to generate expectancies about the where and when of the middle target sequence element by interpolating the space-time trajectory implied by the fixed spatiotemporal positions of the initial (A) and final (B) bounding elements. For the kappa effect, when the middle target element $(\mathrm{X})$ violates the constant velocity assumption along the space dimension, perceptual adjustments along the time dimension are required in order to maintain the sense of constant velocity across the display. Conversely, for the tau effect, when the 
middle target element $(\mathrm{X})$ violates the constant velocity assumption along the time dimension, perceptual adjustments along the space dimension are required in order to maintain a constant velocity. From this perspective, the critical independent variable with respect to the kappa and tau effects is velocity $(\Delta s / \Delta t)$, rather than absolute temporal or spatial separation of the sequence elements.

To provide a formal quantitative account of kappa and tau effects in the visual domain, B. Jones and Huang (1982; Huang \& B. Jones, 1982; see also Anderson, 1974) developed an imputed velocity model. In the kappa version of this model, judgments about the relative timing of sequence elements are considered to be a weighted combination of the objective timing of the target element and an expectation about target timing given constant velocity and target spacing. The model is formalized as follows:

$$
P(B)=f[w t+(1-w) E(t)] .
$$

In Equation $1, P(B)$ refers to the probability that individuals will judge the target tone to be closer in time to the final element, B, corresponding to a long-short response. The variable $t$ refers to the objective timing of the middle target element relative to the first bounding element (denoted t 1 in Figure 1); $E(t)$ then represents the expected value of $t 1$. The expected timing, $E(t)$, is determined by dividing $\Delta s$ (the distance corresponding to $\mathrm{s} 1$ in Figure 1) by imputed velocity, $V: E(t)=\Delta s / V$; this equation follows from algebraic manipulation of the equation for velocity, $V=\Delta s / \Delta t$. The function $f$ is a sigmoid function that maps the perceived target timing to a probability of a response. The weight parameter, $w \in[0,1]$, specifies the relative contribution of $t$ and $E(t)$ to participants' judgments about element timing. When $w=1$, the objective timing of the target is the sole contributor to perceived timing of the target, and there is no kappa effect. However, when $w<1$, the perceived target timing is based partially on the expected target timing derived from imputed velocity, and a kappa effect is observed. Smaller estimated values of $w$ correspond to larger kappa effects. In sum, in the imputed velocity model of the kappa effect, perceived target timing in the canonical kappa task is based on a combination of objective timing and expected timing; the expected timing of the target is derived from imputed velocity and objective stimulus spacing.

In the tau version of the imputed velocity model, judgments about the relative spacing of sequence elements are considered to be a weighted combination of the objective spacing of the target element and an expectation about the distance traveled to the target location given constant velocity and travel time (Huang \& B. Jones, 1982). The model is formalized as follows:

$$
P(B)=f[w s+(1-w) E(s)] .
$$

In Equation 2, $P(B)$ refers to the probability that individuals will judge the target tone to be closer in space to the final element, B. The variable $s$ refers to the objective spacing of the middle target element, relative to the first bounding element (denoted s1 in Figure 1); $E(s)$ then represents the expected value of s1. The expected spacing, $E(s)$, is determined by multiplying the travel time of the target, $\Delta t$ (denoted $\mathrm{t} 1$ in Figure 1), by imputed velocity, $V: E(s)=\Delta t * V$; this equation follows from algebraic manipulation of the equation for velocity, $V=\Delta s / \Delta t$. The function $f$ similarly maps the perceived target spacing to a probability of a response. The weight parameter, $w \in[0,1]$, specifies the relative contribution of $s$ and $E(s)$ to participants' judgments about element spacing. When $w=1$, the objective spacing of the target is the sole contributor to perceived spatial location of the target, and there is no tau effect. However, when $w<1$, the perceived spatial location of the target stimulus is based partially on the expected target location derived from imputed velocity, and a tau effect is observed. Smaller estimated values of $w$ correspond to larger tau effects. In sum, in the imputed velocity model of the tau effect, the perceived target spacing in the canonical tau task is based on a combination of objective spacing and expected spacing; the expected spacing of the target is derived from imputed velocity and objective stimulus timing.

Data on the effects of velocity on the magnitude of the kappa and tau effects are mixed. Huang and B. Jones (1982; see also B. Jones \& Huang, 1982) provided initial support for the imputed velocity model and reported that velocity had opposite effects on the magnitude of kappa and tau (as indexed by $w$ ). Huang and B. Jones varied the velocity of eight-element sequences and had participants judge either the temporal extent (kappa version) or the spatial extent (tau version) of the final interval. For the kappa version, the participants placed the final interval into one of five duration categories, whereas for the tau version, the participants placed the interval into one of five distance categories; the participants were trained on all duration and distance categories prior to testing. Velocity varied from a relatively fast value of $8^{\circ} / 160 \mathrm{msec}$ to a relatively slow value of $8^{\circ} / 1,500 \mathrm{msec}$ (where distance was given in degrees of visual angle subtended by the display). For kappa, relatively large $w$ values (weak kappa effects) were reported at relatively slow velocities, whereas smaller $w$ values, associated with stronger kappa effects, were reported as velocity increased. Conversely, for tau, relatively small $w$ values (strong tau effects) were reported at relatively slow velocities, whereas larger $w$ values, associated with weaker tau effects, were reported as velocity increased. The finding for tau, in particular, differs from that in Bill and Teft (1972), who, using an AXB design and the canonical task, observed stronger visual tau effects at faster velocities.

To explain the opposite effect of velocity on the magnitude of the kappa and tau effects, Huang and B. Jones (1982) proposed that when there is uncertainty about the magnitude of the to-be-attended dimension (as when the task becomes more difficult), the observer may be forced to base his/her judgment on the to-be-ignored dimension. In this respect, Huang and B. Jones proposed that temporal judgments become more difficult at faster presentation rates; thus, observers should be forced to rely on element spacing as a cue at relatively fast velocities, and there should be a larger kappa effect. Conversely, under the assumption that memory for spatial location of visual targets deteriorates over time and, thus, is worse at slower presentation rates, observers should be forced to rely on element timing as a cue to spatial location at relatively slow velocities, and there should thus be a larger tau effect. 


\section{Auditory Kappa and Tau Effects: Theory and Data}

Studies examining kappa and tau effects in the auditory domain have, for the most part, varied the timing and pitch spacing of sequences of sounds (Cohen et al., 1954; Crowder \& Neath, 1995; Henry \& McAuley, 2009a; MacKenzie, 2007; Shigeno, 1986, 1993; Yoblick \& Salvendy, 1970), rather than the separation of the sounds in physical space (see Grondin \& Plourde [2007] and Sarrazin, Giraudo, \& Pittenger [2007] for two exceptions). As in the visual domain, canonical auditory kappa and tau tasks involve an AXB design; a series of three tones is presented, and the pitch and timing of the first and third (bounding) tones (A and B) are fixed, whereas the pitch and timing of the middle target tone $(\mathrm{X})$ vary, relative to the pitch and temporal bisection points of the bounding stimuli. Thus, the three-tone sequence marks out two pitch intervals ( $\mathrm{p} 1, \mathrm{p} 2)$ and two temporal intervals (t1, t2), with the pitch and temporal bisection points corresponding to $\mathrm{p} 1=\mathrm{p} 2$ and $\mathrm{t} 1=\mathrm{t} 2$, respectively.

Analogous to visual kappa, observers in auditory kappa tasks typically judge whether the target tone is closer in time to either the first bounding element (A) or the third bounding element (B) by making a binary short-long versus long-short decision about the temporal pattern of the three-tone sequence or by explicitly adjusting the timing of the target element so that it subjectively bisects the time interval marked by the bounding elements. In this case, observers tend to make systematic errors such that, when $\mathrm{p} 1<\mathrm{p} 2$, the pattern of durations is perceived as short-long $(\mathrm{t} 1<\mathrm{t} 2)$, even when the objective pattern of durations is long-short $(\mathrm{t} 1>\mathrm{t} 2)$. Similarly, when $\mathrm{p} 1>\mathrm{p} 2$, observers tend to perceive the pattern of durations as long-short (t1 > t2) (Cohen et al., 1954; Shigeno, 1986, 1993). Conversely, for auditory tau, observers typically judge whether the target element is closer in pitch to either the first or the third bounding element by making a binary closer to $A$ versus closer to $B$ decision or by explicitly adjusting the pitch of the target element so that it subjectively bisects the pitch distance marked by the bounding elements. In this case, observers tend to make systematic errors such that, when $\mathrm{t} 1<\mathrm{t} 2$, the target is perceived as closer to $\mathrm{A}(\mathrm{p} 1<\mathrm{p} 2)$, even when the target is objectively closer to $\mathrm{B}(\mathrm{p} 2>\mathrm{p} 1)$. Similarly, when $\mathrm{t} 1>\mathrm{t} 2$, observers tend to perceive that the target is closer to B ( $1>$ p2) (Christensen \& Huang, 1979; Cohen et al., 1974; Shigeno, 1986, 1993).

Henry and McAuley (2009a) showed that the same principles used to develop an imputed velocity model of the visual kappa and tau effects can also be used to develop a model of the auditory kappa and tau effects. In the case of variations in pitch spacing, velocity can be measured as change in pitch per change in unit time, $V_{p}=\Delta p / \Delta t$, where $\Delta p$ is expressed in semitones (STs; a musical interval corresponding to the frequency ratio $\Delta f / f=1.059$ ). Thus, the model of the auditory kappa effect is given by

$$
P(B)=f[w t+(1-w) E(t)] .
$$

The perceived timing of the target tone is assumed to be based on a weighted combination of the objective timing of the target, $t$, and the expected timing, $E(t)$, derived from im- puted pitch velocity and target pitch, $E(t)=\Delta p / V_{p}$. For auditory tau, the perceived pitch of the target tone is assumed to be based on a weighted combination of the pitch of the target, $p$, and the expected pitch, $E(p)$, derived from imputed pitch velocity, $V_{p}$, and the temporal interval between the onset of the first bounding tone (A) and the onset of the target tone, $E(p)=\Delta t * V_{p}$. For the model of the tau effect, the equation relating objective target pitch and expected pitch to a closer to $B$ response proportion is given by

$$
P(B)=f[w p+(1-w) E(p)] .
$$

Analogous to visual tau, the weight parameter, $w$, estimates the relative contributions of $p$ and $E(p)$ to perceived target pitch, with $f$ given by a sigmoid function and smaller values of $w$ associated with larger tau effects.

To test an imputed pitch velocity model, Henry and McAuley (2009a) examined the effect of velocity on the magnitude of the auditory kappa effect. Using the canonical task, fast ( $8 \mathrm{ST} / 728 \mathrm{msec})$, medium $(8 \mathrm{ST} / 1,000 \mathrm{msec})$, and slow $(8 \mathrm{ST} / 1,600 \mathrm{msec})$ velocities were tested for both ascending and descending tone sequences. Overall, the auditory version of the imputed velocity model proposed by the authors provided good quantitative fits to participants' judgments about target timing. With respect to velocity, the auditory kappa effect was found to be strongest for velocities that fell at the upper (fast) end of the tested range $(8 \mathrm{ST} / 728 \mathrm{msec})$ and weakest for the slowest (8 ST/1,600 msec) tested velocity for both ascending and descending sequences; average values of $w$ obtained from model fits ranging from .86 in the fast velocity condition to .97 in the slow velocity condition.

This result is notably consistent with an auditory motion hypothesis (M. R. Jones, 1976; M. R. Jones \& Yee, 1993; MacKenzie, 2007), which proposes that interactions between perceived pitch and time in kappa and tau tasks are the result of an interpolation of a pitch-time trajectory implying constant velocity that occurs within a range of pitch velocities that promote attention to motion-like properties of auditory sequences. Critically, as pitch velocity increases within this range, attention to motion-like properties is expected to increase and produce larger kappa and tau effects. This result is also consistent with the view proposed by Huang and B. Jones (1982) that, at faster presentation rates, judgments about target timing should become increasingly difficult and, thus, resemble more closely the pitch spacing of the sequence, resulting in stronger kappa effects. These two hypotheses make different predictions, however, for the auditory tau effect. The auditory motion hypothesis predicts that increasing pitch velocity should increase the magnitude of the auditory tau effect, as is found for auditory kappa. However, the hypothesis of Huang and B. Jones predicts that velocity will have the opposite effect on auditory tau. That is, increasing pitch velocity should weaken, rather than strengthen, the auditory tau effect. Some initial support for an auditory motion interpretation of the auditory tau effect has come from Cohen et al. (1954), who used the method of adjustment, and Shigeno (1993), who used the canonical tau task and an AXB design and observed that the auditory tau effect is larger when the pitch distance 
spanned by the bounding tones is increased, while holding presentation rate constant.

The present study further distinguishes these two hypotheses by examining the effect of pitch velocity on the magnitude of the auditory tau effect by varying the temporal separation of the bounding tones, while holding the pitch separation of the bounding tones constant, in both ascending and descending sequences, using an AXB paradigm (Shigeno, 1986, 1993).

\section{Overview}

The goals of the present study were twofold: (1) to evaluate an imputed pitch velocity model of the auditory tau effect and (2) to examine the contribution of velocity to the magnitude of the effect in order to provide a further test of an auditory motion hypothesis. Two experiments were conducted using an AXB design and the canonical auditory tau task. Participants in both experiments listened to either three-tone ascending or descending sequences and indicated whether the middle target tone was closer in pitch to the first tone (closer to $A$ ) or to the third tone (closer to $B$ ), ignoring any variations in target timing. The separation of the bounding elements was fixed at $16 \mathrm{ST}$, yielding a bisection point of $8 \mathrm{ST}$ for the middle target tone. The time interval between bounding tone onsets took on values of $T=728,1,000$, and 1,600 msec, yielding three velocity conditions: fast (16 ST/728 msec), medium (16 ST/1,000 msec), and slow (16 ST/1,600 msec). Velocity conditions were equally spaced in units of $\mathrm{ST} / \mathrm{sec}$ on the basis of evidence suggesting that magnitude estimates of velocity increase linearly as a function of ST/sec (Henry \& McAuley, 2009b). Because previous research on auditory tau has revealed relatively weaker effects, as compared with auditory kappa (Cohen et al., 1954; Shigeno, 1993), the pitch separation (in semitones) between bounding tones for the three velocity conditions was larger than the separation used in previous research on auditory kappa (Henry \& McAuley, 2009a).

Experiment 1 was a baseline study that examined participants' ability to detect deviations in the pitch of the middle target tone from the 8-ST bisection point of sequences that were always isochronous, thus instantiating a constant velocity. Experiment 2 examined the same three velocity conditions but included variable target timing, thus introducing deviations from constant velocity. If the results of Henry and McAuley (2009a) for the auditory kappa effect generalize to the auditory tau effect in a manner consistent with an auditory motion hypothesis, the magnitude of the auditory tau effect should be largest at the fastest tested velocity (16 ST/728 msec) and smallest at the slowest tested velocity (16 ST/1,600 msec) for both ascending and descending sequence conditions. However, if the auditory tau effect is attributable to a deterioration of the memory of space (pitch) at relatively slow presentation rates and, thus, a stronger reliance on sequence timing as a cue to location, the magnitude of the tau effect should be largest at the slowest tested velocity $(16 \mathrm{ST} / 1,600 \mathrm{msec})$ and smallest at the fastest tested velocity (16 ST/728 msec) for both ascending and descending sequence conditions.

\section{EXPERIMENT 1}

\section{Method}

Design. Experiment 1 implemented a 2 (direction: ascending, descending) $\times 3$ (velocity: fast, medium, slow) $\times 8$ (target pitch level) mixed factorial design. Velocity and direction were varied between subjects, whereas target pitch level was varied within subjects.

Participants. Forty-six Bowling Green State University undergraduates between the ages of 18 and 45 years ( 25 of them female)
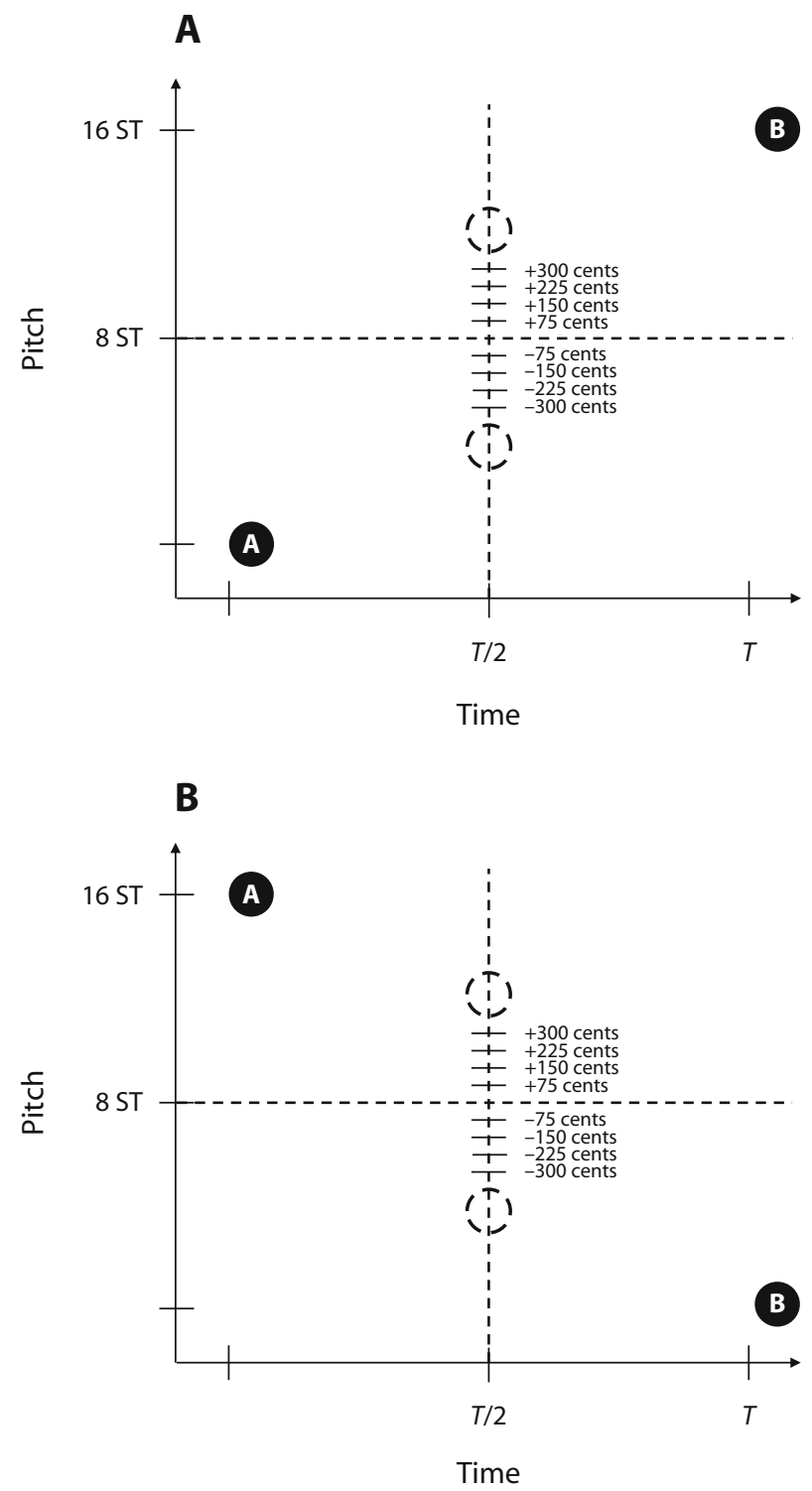

Figure 2. Task diagram for Experiment 1 (isochronous timing) for (A) ascending sequences and (B) descending sequences. The pitch separation of the bounding tones was 16 semitones (STs), yielding fast (16 ST/728 msec), medium (16 ST/1,000 msec), and slow (16 ST/1,600 msec) velocity conditions; the position of the target tone (open circles) in pitch space was varied from trial to trial and took on values that were $\pm 75, \pm 150, \pm 225$, or \pm 300 cents, relative to the 8-ST bisection point of the bounding tones. In Experiment 1, target tone timing was held constant such that all sequences had isochronous timing. 
participated in return for a cash payment of $\$ 5$ or course credit. All the participants self-reported normal hearing and had a range of formal musical training $(M=4.38$ years, $S D=4.25)$. The participants heard either ascending or descending sequences and were randomly assigned to the fast $\left(16 \mathrm{ST} / 528 \mathrm{msec} ; n_{\mathrm{Asc}}=7, n_{\text {Desc }}=8\right)$, medium $\left(16 \mathrm{ST} / 800 \mathrm{msec} ; n_{\mathrm{Asc}}=7, n_{\text {Desc }}=8\right)$, or slow $(16 \mathrm{ST} / 1,400 \mathrm{msec}$; $n_{\text {Asc }}=7, n_{\text {Desc }}=9$ ) velocity condition.

Stimuli and equipment. The stimuli were three-tone sequences consisting of 100-msec pure tones that ascended or descended in pitch (see Figure 2). For ascending sequences, the fundamental frequencies of the first and third tones were always 329.6 and $830.6 \mathrm{~Hz}$ (the musical notes $\mathrm{E}_{4}$ and $\mathrm{A}_{5}$-flat, respectively); for descending sequences, the fundamental frequencies of the first and third tones were always $830.6 \mathrm{~Hz}\left(\mathrm{~A}_{5}\right.$-flat) and $329.6 \mathrm{~Hz}\left(\mathrm{E}_{4}\right)$. This produced a 16-ST separation between the first and third (bounding) tones. In the fast, medium, and slow velocity conditions, the temporal interval between onsets of the bounding tones was $T=728,1,000$, and 1,600 msec, respectively. Sequence timing was always isochronous, with the middle target tone onset always occurring at the temporal bisection point: $T / 2=364,500$, or $800 \mathrm{msec}$, depending on velocity condition. There were eight possible frequency values for the target tone: $440 \mathrm{~Hz}\left(\mathrm{~A}_{4}\right)$, $459.5 \mathrm{~Hz}\left(\mathrm{~A}_{4}+75\right), 479.8 \mathrm{~Hz}\left(\mathrm{~B}_{4}\right.$-flat +50$), 501.1 \mathrm{~Hz}\left(\mathrm{~B}_{4}+25\right)$, $546.4 \mathrm{~Hz}\left(\mathrm{C}_{5}+75\right), 570.6 \mathrm{~Hz}\left(\mathrm{C}_{5}\right.$-sharp +50$), 595.9 \mathrm{~Hz}\left(\mathrm{D}_{5}+25\right)$, $622.3 \mathrm{~Hz}$ ( $\mathrm{E}_{5}$-flat). These values corresponded to deviations of \pm 75 , $\pm 150, \pm 225$, and \pm 300 cents relative to the 8 -ST bisection point of each sequence, where 100 cents $=1 \mathrm{ST}$.

The stimuli were generated using Audacity, Version 1.2.6, for Microsoft Windows; stimulus presentation and response collection were controlled by E-Prime 1.2 software (Psychology Software Tools, Inc.). Tone sequences were presented at a comfortable listening level $(\approx 70 \mathrm{~dB})$ through Sennheiser HD 280 Pro headphones. Responses were made by pressing one of two horizontally aligned buttons on a response box; closer to $A$ responses were made by pressing the left button, and closer to $B$ responses were made by pressing the right button.

Procedure. The participants were instructed that for each threetone sequence, the middle tone would be closer in pitch to either the first or the third tone, referred to as $A$ and $B$, respectively. The participants were asked to respond closer to $A$ when they thought the target tone was closer in pitch to the first tone and to respond closer to $B$ when they thought the target tone was closer in pitch to the third tone. The participants first completed a short (eight-trial) familiarization block with feedback, followed by five test blocks without feedback; familiarization trials consisted of either ascending or descending sequences (depending on sequence condition), with deviations in target pitch level that were \pm 600 or \pm 700 cents. Test blocks consisted of 40 trials. Within a test block, the pitch of the middle target tone varied from trial to trial; the participants heard each of the eight target pitch levels five times within a block (with 25 total judgments about each target pitch level across the five blocks). The entire experiment lasted approximately $30 \mathrm{~min}$.

Data analysis. Proportions of closer to $B$ responses for ascending sequences and descending sequences were determined for each participant for each of the eight target pitch levels, averaged over the five test blocks. Pitch discrimination thresholds (just noticeable differences [JNDs]) and points of subjective equality (PSEs) were then estimated from the resulting psychometric curves for each participant by first transforming response proportions to $z$ scores and then using linear regression (see Macmillan \& Creelman, 1991, pp. 219-220). Using this method, JND estimates half the distance between the 25th and 75th percentiles of the cumulative response function and PSE estimates the median of the curve, corresponding to the pitch value (in cents) of the target tone judged closer to B $50 \%$ of the time (the subjective pitch bisection point). PSE was converted to a directional constant error (CE), where negative $\mathrm{CE}$ values indicated underestimation of the 8-ST bisection point and positive CE values indicated overestimation of the 8-ST bisection point. All CE and JND values are reported in cents.

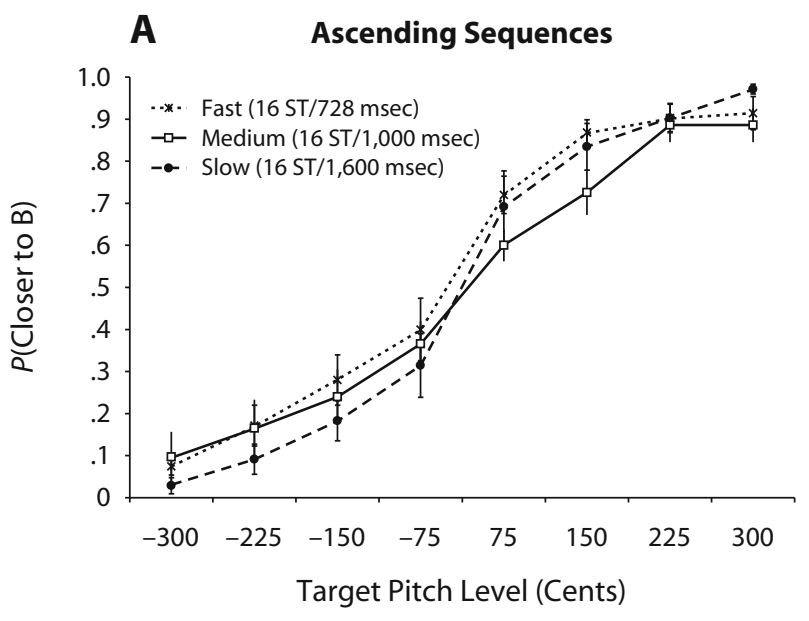

B

Descending Sequences

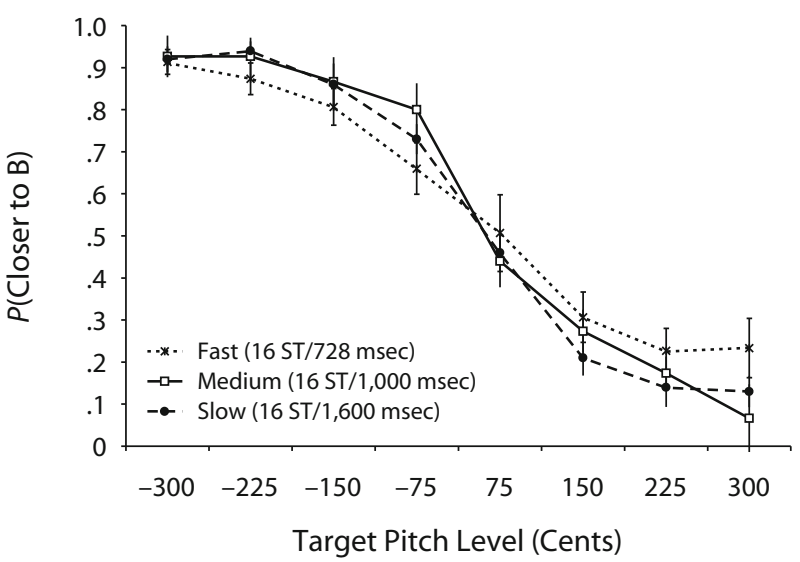

Figure 3. Experiment 1: Aggregate psychometric curves with standard error bars for the fast $(16 \mathrm{ST} / 728 \mathrm{msec})$, medium (16 ST/1,000 msec), and slow (16 ST/1,600 msec) velocity conditions for ascending (A) and descending (B) sequences.

\section{Results and Discussion}

Figure 3 shows the aggregate psychophysical curves with standard error bars for the fast, medium, and slow velocity conditions for ascending sequences (panel A) and descending sequences (panel B). ${ }^{1}$ A 3 (velocity) $\times 2$ (direction) between-subjects ANOVA on CE scores revealed no effect of velocity $[F(2,40)=0.60, p=.56]$ but a marginally significant effect of direction $[F(1,40)=3.53$, $\left.p=.07, \eta_{\mathrm{p}}^{2}=.08\right]$. Mean CE scores were slightly higher for descending sequences $(M=49.9 \pm 12.5$ cents $)$ than for ascending sequences ( $M=15.2 \pm 13.6$ cents $)$. Singlesample $t$ tests revealed that CEs for descending sequences, but not for ascending sequences, were significantly greater than zero $[t(24)=3.68, p<.01]$. There was no interaction between velocity and direction $[F(2,40)=0.05, p=$ $.95]$. On average, JNDs for pitch were $155.5 \pm 14.2$ cents and did not vary as a function of velocity $[F(2,40)=$ $0.82, p=.45]$ or direction $[F(1,40)=0.10, p=.75]$. The velocity $\times$ direction interaction also was not significant $[F(2,40)=1.23, p=.30]$. 
Overall, Experiment 1 shows that for isochronous sequences, the subjective pitch bisection point does not vary as a function of velocity and differs only slightly from the objective 8-ST value. Larger deviations were found for descending sequences than for ascending sequences, where the 8-ST bisection point was generally overestimated in all three velocity conditions by approximately the same amount. Hubbard (1995, Experiments 1, 3, and 4) reported a similar asymmetry such that forward displacement of a target tone along a descending trajectory was larger than forward displacement along an ascending trajectory. Pitch discrimination thresholds for Experiment 1 were somewhat larger than those observed for same-different pitch discrimination tasks involving two-tone sequences (e.g., Johnsrude, Penhune, \& Zatorre, 2000) but were similar in magnitude to those in studies using an AXB design (Shigeno, 1986). For ascending three-tone sequences, Shigeno (1986) obtained an average JND of approximately 160 cents, which is comparable to the average 155 -cent value reported here.

A second experiment examined the same three velocity conditions for ascending and descending sequence conditions but, additionally, varied the timing of the target tone from trial to trial in order to examine the effect of pitch velocity on the magnitude of the auditory tau effect.

\section{EXPERIMENT 2}

\section{Method}

Design. Experiment 2 implemented a 2 (direction: ascending, descending) $\times 3$ (velocity: fast, medium, slow) $\times 8$ (target pitch level) $\times 5$ (target time level) mixed factorial design. Velocity and direction were varied between subjects, whereas target pitch level and target time level were varied within subjects. For ascending and descending conditions, the three pitch velocity conditions ( $16 \mathrm{ST} / 728 \mathrm{msec}$ [fast], $16 \mathrm{ST} / 1,000 \mathrm{msec}$ [medium], $16 \mathrm{ST} / 1,600 \mathrm{msec}$ [slow]) and eight target pitch levels $( \pm 75, \pm 150, \pm 225$, or \pm 300 cents, relative to the 8 -ST bisection point) from Experiment 1 were crossed with five target time levels $(-66 \%,-33 \%, 0 \%, 33 \%$, or $66 \%$, relative to the temporal bisection point (T/2) of the bounding tone onsets).

Participants. One hundred five Bowling Green State University undergraduates between the ages of 18 and 35 years ( 59 of them female) participated in return for course credit. ${ }^{2}$ All the participants self-reported normal hearing and had a range of formal musical training $(M=3.09$ years, $S D=3.39)$. The participants were randomly assigned to the fast $\left(16 \mathrm{ST} / 728 \mathrm{msec} ; n_{\mathrm{Asc}}=17, n_{\text {Desc }}=17\right)$, medium $\left(16 \mathrm{ST} / 1,000 \mathrm{msec} ; n_{\mathrm{Asc}}=14, n_{\text {Desc }}=18\right)$, or slow $(16 \mathrm{ST} / 1,600 \mathrm{msec}$; $n_{\text {Asc }}=20, n_{\text {Desc }}=19$ ) velocity condition.

Stimuli and equipment. The stimuli and equipment were the same as those in Experiment 1, with the exception that the temporal position of the middle target tone took on values of $-66 \%,-33 \%$, $0 \%,+33 \%$, and $+66 \%$, relative to the temporal bisection point of the sequence $(T / 2=364,500$, or $800 \mathrm{msec})$; see Figure 4 .

Procedure. The procedure for Experiment 2 was similar to that in Experiment 1. The participants first completed a short (eighttrial) familiarization block with feedback, followed by a series of test blocks without feedback; familiarization trials consisted of either ascending or descending sequences, with deviations in target pitch level that were \pm 600 or \pm 700 cents. Following familiarization, the participants were informed that for each three-tone sequence, the temporal position of the middle target tone would vary from trial to trial. Critically, the participants were instructed to ignore variations in timing and judge only the pitch of the target tone. There were 10 test blocks with 40 trials per block. Within a test block, the par-
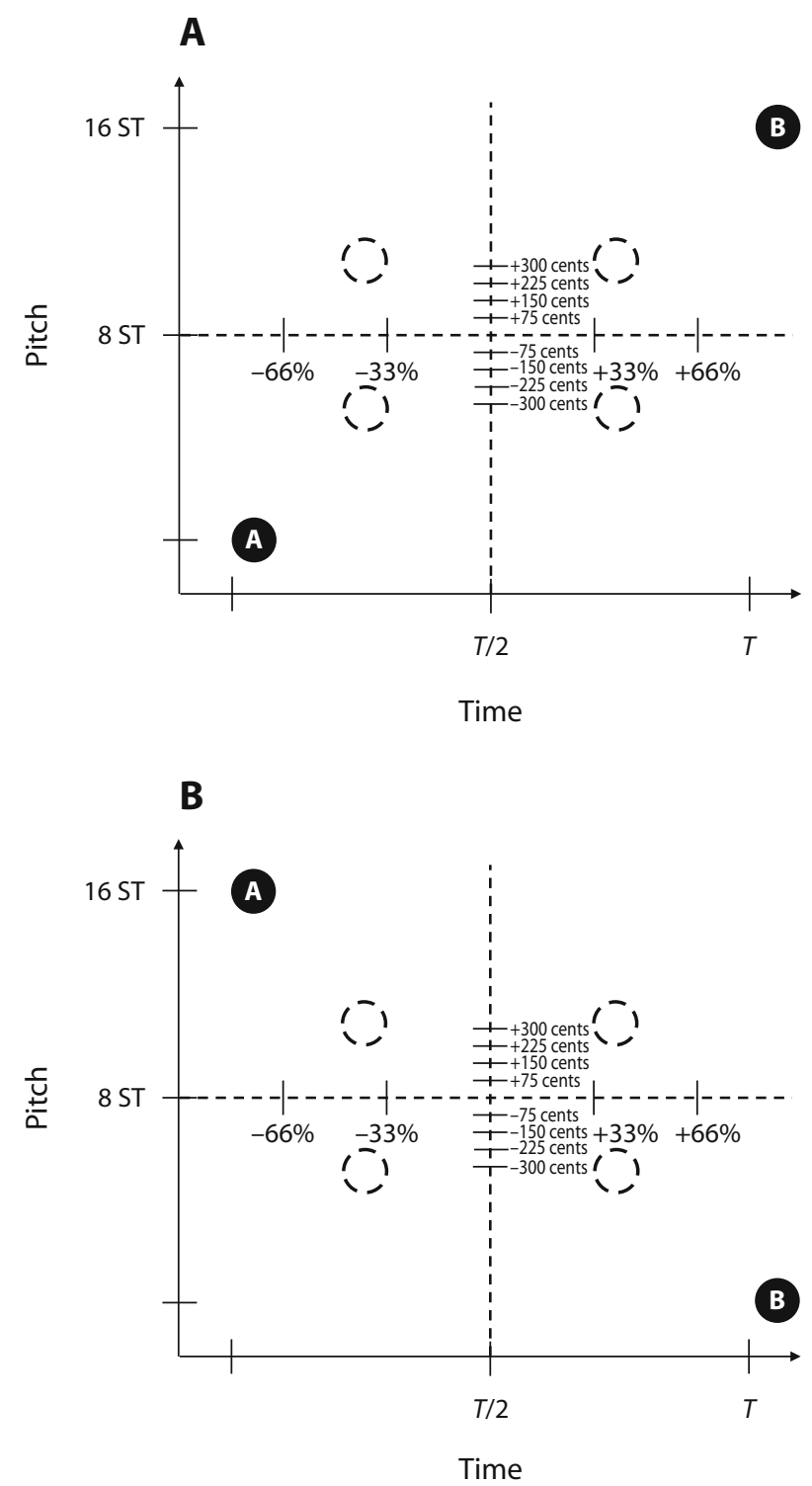

Figure 4. Task diagram for Experiment 2 (variable timing) for ascending (A) and descending (B) sequences. The pitch separation of the bounding tones was 16 semitones (STs), yielding fast (16 ST/728 msec), medium (16 ST/1,000 msec), and slow (16 ST/1,600 msec) velocity conditions; the position of the target tone (open circles) in pitch space was varied from trial to trial and took on values that were $\pm 75, \pm 150, \pm 225$, or \pm 300 cents, relative to the pitch bisection point of the bounding tones (8 ST). In Experiment 2 , target tone timing was variable and took on values of $-66 \%,-33 \%, 0 \%,+33 \%$, and $+66 \%$, relative to the temporal bisection point of the bounding tones $(T / 2)$.

ticipants heard each of the 8 target pitch level and 5 target time level combinations once, yielding 10 responses for each of the 40 combinations over the course of the experiment. The entire experiment lasted approximately $60 \mathrm{~min}$.

\section{Results and Discussion}

To evaluate the effect of pitch velocity and sequence direction on the magnitude of the auditory tau effect, a 3 (velocity) $\times 2$ (direction) $\times 5$ (target time level) mixed 

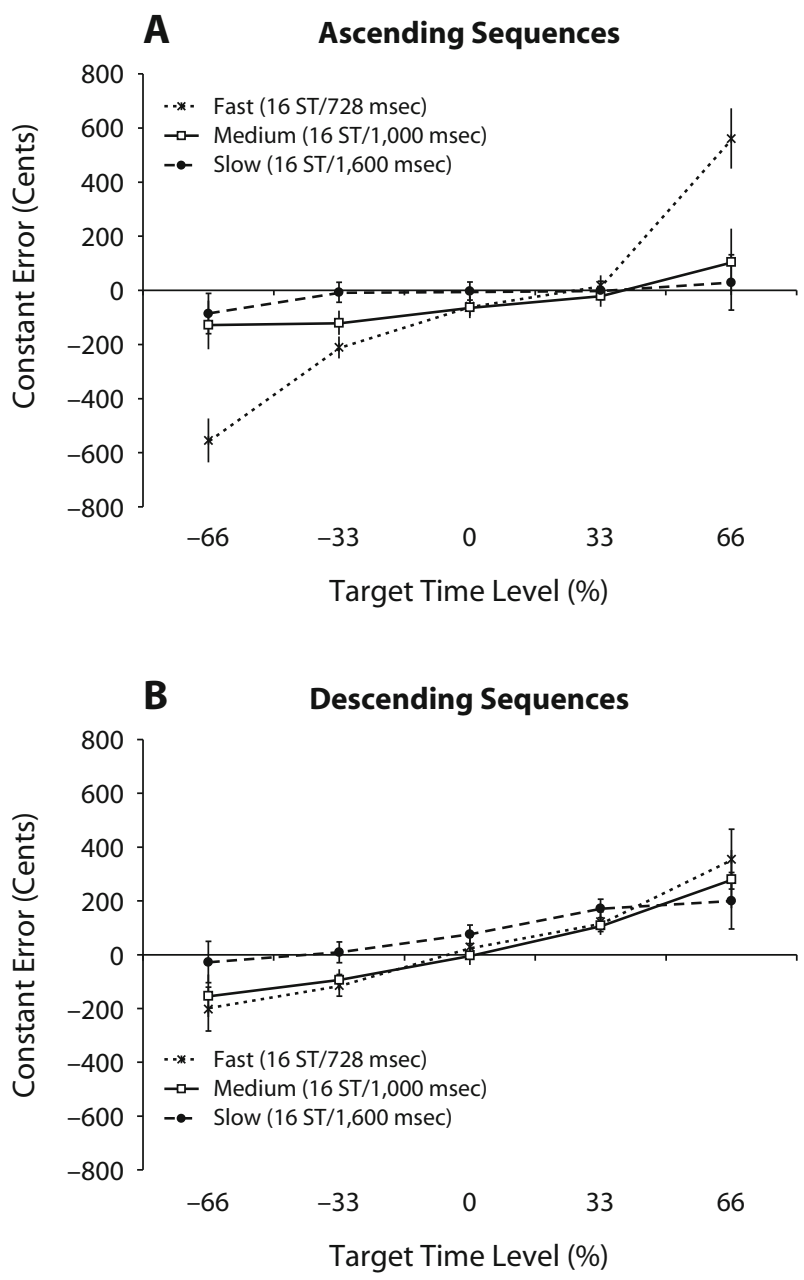

Figure 5. Experiment 2: Mean constant error in cents (with standard error bars) as a function of target time level for the fast, medium, and slow velocity conditions for ascending (A) and descending (B) sequences.

measures ANOVA was conducted on CE scores. Sphericity was violated for target time level $(p<.001)$, so all main effects and interactions involving target time level are reported with degrees of freedom adjusted according to a Greenhouse-Geisser correction. CE data for ascending and descending sequences are summarized in Figure 5. The ANOVA revealed a main effect of target time level $\left[F(1.77,175.45)=40.01, p<.001, \eta_{\mathrm{p}}^{2}=.29\right]$ and, critically, a significant target time level $\times$ velocity inter- action $\left[F(3.54,175.45)=8.08, p<.001, \eta_{\mathrm{p}}^{2}=.14\right]$. The effect of target time level was indicative of an auditory tau effect; judgments about the pitch of the target tone were systematically distorted in the direction of deviations in target timing. When the target tone occurred earlier than the temporal bisection point $(\mathrm{t} 1<\mathrm{t} 2), \mathrm{CE}$ scores were generally negative, indicating that the listeners were more likely to respond that the target tone was closer in pitch to the first tone (A) than to the third tone (B). Conversely, when the target tone occurred after the temporal bisection point $(\mathrm{t} 1>\mathrm{t} 2)$, CE scores were generally positive, indicating that the participants were more likely to respond that the target tone was closer in pitch to the third tone (B) than to the first tone (A). The interaction between target time level and velocity revealed greater distortions in perceived target pitch at faster velocities, supporting the auditory motion hypothesis.

The main effect of direction was also significant $\left[F(1,99)=9.08, p<.01, \eta_{\mathrm{p}}^{2}=.08\right]$; mean CEs $( \pm S E M)$ for ascending and descending sequences were -35.7 $( \pm 20.7)$ and $50.8( \pm 19.9)$ cents, respectively. As in Experiment 1 , individual single-sample $t$ tests revealed that mean CE for descending sequences, but not for ascending sequences, was greater than zero $[t(53)=3.46, p<$ $.01]$. The three-way target tone time $\times$ velocity $\times$ direction interaction was significant $[F(3.54,175.45)=2.65, p<$ $\left..05, \eta_{\mathrm{p}}^{2}=.05\right]$. This interaction was driven by a weakened effect of velocity on the magnitude of the auditory tau effect for descending sequences, as compared with ascending sequences. The target tone time $\times$ velocity interaction was significant for ascending sequences $[F(1.74,83.66)=$ $\left.6.82, p<.001, \eta_{\mathrm{p}}^{2}=.22\right]$, but not for descending sequences $\left[F(3.49,89.09)=1.69, p=.17, \eta_{\mathrm{p}}^{2}=.06\right]$. The main effect of velocity $[F(2,99)=1.07, p=.35]$, the velocity $\times$ direction interaction $[F(2,99)=0.07, p=.93]$, and the target time level $\times$ velocity interaction $[F(1.77,175.45)=0.70$, $p=.50]$ failed to reach significance.

Next, the proposed imputed pitch velocity model was fit to each participant's response proportions in order to use estimates of $w$ to more formally quantify the degree to which participants' judgments were based on expected pitch for each of the three velocity conditions. Recall that smaller values of $w$ correspond to larger tau effects. Model fits minimized the root mean square error of approximation (RMSE_A) between the observed and the predicted response proportions at each of the eight target pitch levels $( \pm 75, \pm 150, \pm 225$, or \pm 300 cents $)$ for each of the five target time levels $(-66 \%,-33 \%, 0 \%,+33 \%$,

Table 1

Values of $w( \pm S E M)$ Estimated Using the Imputed Velocity Model for the Fast, Medium, and Slow Velocity Conditions, Ascending and Descending Sequences, for Experiment 2

\begin{tabular}{|c|c|c|c|c|c|c|}
\hline \multirow[b]{3}{*}{ Direction } & \multicolumn{6}{|c|}{ Velocity Condition } \\
\hline & \multicolumn{2}{|c|}{$\begin{array}{c}\text { Fast } \\
(16 \mathrm{ST} / 728 \mathrm{msec})\end{array}$} & \multicolumn{2}{|c|}{$\begin{array}{c}\text { Medium } \\
(16 \mathrm{ST} / 1,000 \mathrm{msec})\end{array}$} & \multicolumn{2}{|c|}{$\begin{array}{c}\text { Slow } \\
(16 \mathrm{ST} / 1,600 \mathrm{msec})\end{array}$} \\
\hline & JND & SEM & JND & $\overline{S E M}$ & JND & SEM \\
\hline Ascending & .72 & .031 & .86 & .034 & .93 & .029 \\
\hline Descending & .76 & .031 & .76 & .030 & .83 & .029 \\
\hline
\end{tabular}

Note—ST, semitone; JND, just noticeable difference. 
Table 2

Estimated Just Noticeable Differences (JNDs; $\pm S E M$ s) Reported in Cents

for Each of Five Pitch Levels of the Target Tone for the Fast, Medium, and Slow Velocity Conditions in Experiment 2

\begin{tabular}{|c|c|c|c|c|c|c|}
\hline \multirow{3}{*}{$\begin{array}{l}\text { Target } \\
\text { Time } \\
\text { Level }\end{array}$} & \multicolumn{6}{|c|}{ Velocity Condition } \\
\hline & \multicolumn{2}{|c|}{$\begin{array}{c}\text { Fast } \\
(16 \mathrm{ST} / 728 \mathrm{msec})\end{array}$} & \multicolumn{2}{|c|}{$\begin{array}{c}\text { Medium } \\
(16 \mathrm{ST} / 1,000 \mathrm{msec})\end{array}$} & \multicolumn{2}{|c|}{$\begin{array}{c}\text { Slow } \\
(16 \mathrm{ST} / 1,600 \mathrm{msec})\end{array}$} \\
\hline & JND & $S E M$ & JND & $S E M$ & JND & $S E M$ \\
\hline$-66 \%$ & 340 & 47 & 191 & 49 & 256 & 44 \\
\hline$-33 \%$ & 251 & 27 & 207 & 28 & 197 & 26 \\
\hline $0 \%$ & 217 & 17 & 186 & 17 & 157 & 16 \\
\hline$+33 \%$ & 218 & 45 & 182 & 18 & 170 & 16 \\
\hline$+66 \%$ & 434 & 49 & 229 & 47 & 187 & 42 \\
\hline
\end{tabular}

Note-ST, semitone

and $+66 \%$ ), using a single value of $w$ for each participant. Mean $w$ estimates with standard error values are reported in Table 1 for ascending and descending sequences for the fast, medium, and slow velocity conditions. In general, the imputed velocity model provided reasonable quantitative fits to participant data (mean RMSE_A $=0.16 \pm 0.04$ ); these fits were slightly worse, however, than those previously observed for the auditory kappa effect (Henry \& McAuley, 2009a); see the Appendix for details of model fits and for comparison with auditory kappa.

A 3 (velocity) $\times 2$ (direction) between-subjects ANOVA on $w$ values revealed a main effect of velocity $\left[F(2,99)=10.61, p<.001, \eta_{\mathrm{p}}^{2}=.18\right]$, a main effect of direction $\left[F(1,99)=4.47, p<.05, \eta_{\mathrm{p}}^{2}=.04\right]$, and a significant velocity $\times$ direction interaction $[F(2,99)=3.74$, $\left.p<.05, \eta_{\mathrm{p}}^{2}=.07\right]$. Consistent with the CE data and an auditory motion hypothesis, the magnitude of the auditory tau effect increased with increasing velocity (fast, $M=$ $.70 \pm .03$; medium, $M=.73 \pm .03$; slow, $M=.86 \pm .03$ ) and was somewhat larger for descending sequences $(M=$ $.78 \pm .02)$ than for ascending sequences $(M=.83 \pm$ $.02)$. For the velocity effect, pairwise comparisons using Tukey's HSD indicated reliable differences between the fast and slow velocity conditions $(p<.001)$ and between the medium and slow velocity conditions $(p<.05)$; the difference between the fast and medium velocity conditions failed to reach significance $(p=.14)$. When the interaction between velocity and direction was taken into account, the larger tau effect for descending sequences, as compared with ascending sequences, was found to be reliable for the medium velocity condition $[t(30)=2.37, p<$ $.05]$ and slow velocity condition $[t(37)=3.56, p<.01]$, but not for the fast velocity condition $[t(32)=-0.77, p=$ $.45]$. The correlation between number of years of musical training and $w$ was not significant for either ascending sequences $[r(49)=-.05, p=.71]$ or descending sequences $[r(52)=.01, p=.97]$.

With respect to discrimination thresholds, pitch JNDs were found to be inflated in Experiment $2(M=228.3 \pm$ 11.6 cents), relative to Experiment $1(M=155.5 \pm$ 14.2 cents). A 3 (velocity) $\times 2$ (direction) $\times 5$ (target time level) mixed measures ANOVA was conducted on JNDs. These data are summarized in Table 2. As was found with the analysis of CE scores, sphericity was violated for target time level $(p<.001)$, so the main effect of target time level and interactions with this variable were reported with degrees of freedom adjusted according to a Greenhouse-Geisser correction. The ANOVA revealed a main effect of velocity $\left[F(2,99)=7.60, p<.01, \eta_{\mathrm{p}}^{2}=\right.$ $.13]$, a main effect of target time level $[F(2.45,242.29)=$ $\left.6.40, p<.001, \eta_{\mathrm{p}}^{2}=.06\right]$, and a significant velocity $\times$ target time level interaction $[F(4.90,242.29)=2.89, p<$ $\left..01, \eta_{\mathrm{p}}^{2}=.06\right]$. No other main effects or interactions were significant (all $p \mathrm{~s}>.1$ ).

On average, pitch JNDs were somewhat higher in the fast velocity condition $(M=292.1 \pm 20.3$ cents $)$ than in the medium velocity condition $(M=199.3 \pm 21.0$ cents $)$ or the slow velocity condition $(M=193.5 \pm 18.9$ cents $)$. Pairwise comparisons using Tukey's HSD revealed a reliable difference between JNDs for the fast and medium $(p<.01)$ and fast and slow $(p<.01)$ conditions, but no difference between medium and slow conditions ( $p=$ .97). The effect of target time level revealed that judgments about target pitch were best when the target tone occurred at the temporal bisection point (T/2), which corresponded to isochronous timing; thresholds increased as a function of the temporal distance of the target tone from the bisection point. A trend analysis confirmed that the overall quadratic trend was significant $[F(1,99)=16.64$, $\left.p<.001, \eta_{\mathrm{p}}^{2}=.14\right]$. This quadratic pattern of JNDs as a function of target timing has been termed an expectancy profile (Barnes \& M. R. Jones, 2000; McAuley \& M. R. Jones, 2003). Separate trend analyses for the three velocity conditions indicated that the quadratic trend was significant for the fast velocity condition $[F(1,33)=19.73$, $\left.p<.001, \eta_{\mathrm{p}}^{2}=.37\right]$, but not for the medium $[F(1,31)=$ $0.97, p=.33]$ or slow $[F(1,38)=2.15, p=.13]$ velocity conditions.

In sum, the participants in Experiment 2 showed systematic distortions in perceived pitch as a function of tobe-ignored variations in target timing, indicative of a robust auditory tau effect. Both CE data and estimates of $w$ obtained from fits of the imputed velocity model support the auditory motion hypothesis; the largest tau effect was observed in the fast velocity condition $(16 \mathrm{ST} / 728 \mathrm{msec})$, the smallest tau effect was observed in the slow velocity condition $(16 \mathrm{ST} / 1,600 \mathrm{msec})$, and an intermediate effect was observed in the medium pitch velocity condition $(16 \mathrm{ST} / 1,000 \mathrm{msec})$. Overall, the mediating effect of velocity on the magnitude of the tau effect was some- 
what larger for ascending sequences than for descending sequences. Analysis of $w$ estimates further revealed, however, that the tau effect was generally larger for descending than for ascending sequences, but only for the medium and slow velocities. The magnitude of the tau effect was not correlated with years of musical training. JND data revealed that the participants were somewhat less sensitive overall to target pitch deviations in the fast velocity condition, as compared with the medium and slow velocity conditions. Finally, the participants tended to be better able to discriminate the direction of deviations of the target's pitch from the pitch bisection point when the target tone occurred at the expected location in time (isochronous sequence timing) - specifically, at the fastest velocity.

\section{GENERAL DISCUSSION}

The present study extends an imputed pitch velocity model of the auditory kappa effect proposed by Henry and McAuley (2009a) to the auditory tau effect. Two experiments were conducted involving an AXB design in which listeners judged the relative pitch of a target tone embedded in ascending and descending three-tone sequences. In Experiment 1, we examined isochronous sequences that established constant fast (16 ST/728 msec), medium (16 ST/1,000 msec), and slow (16 ST/1,600 msec) velocity conditions. In Experiment 2, we tested the same three velocity conditions but introduced to-be-ignored variations in the timing of the middle target tone.

Four main findings obtained. First, constant velocity conditions in Experiment 1 revealed no tau effect and pitch discrimination thresholds similar to those found in previous studies involving AXB designs (Shigeno, 1986). Second, to-be-ignored variations in target timing in Experiment 2 revealed a robust auditory tau effect that was mediated by velocity. The magnitude of the auditory tau effect was largest for the fast $(16 \mathrm{ST} / 728 \mathrm{msec})$ velocity, smallest for the slow $(16 \mathrm{ST} / 1,600 \mathrm{msec})$ velocity, and intermediate for the medium $(16 \mathrm{ST} / 1,000 \mathrm{msec})$ velocity for both ascending and descending sequences. Fits of the proposed imputed pitch velocity model revealed a similar pattern, with values of $w$ decreasing with increasing pitch velocity; thus, both the pattern of response proportions and model-based estimates converge to indicate a greater reliance of pitch judgments on target time level at faster velocities. Third, pitch discrimination thresholds in Experiment 2 tended to be lower when the target tone occurred at the temporal bisection point (corresponding to isochronous timing) than when the timing of the target tone deviated from isochrony. Finally, the magnitude of the auditory tau effect tended to be larger for descending sequences than for ascending sequences.

Overall, the results of this study provide support for the proposed imputed velocity model of the auditory tau effect and the associated auditory motion hypothesis. The results, in contrast, do not support the uncertainty hypothesis proposed by Huang and B. Jones (1982), which predicts that increasing velocity should have the opposite effects on the magnitude of tau and kappa; specifically, for tau, the uncertainty hypothesis predicts that values of $w$ should increase, rather than decrease, with increasing velocity.

Lower pitch discrimination thresholds with isochronous timing, as compared with nonisochronous timing, is consistent with previous work by M. R. Jones and colleagues on dynamic attending theory and their view that the observed quadratic pattern reflects enhanced performance for both temporal and nontemporal stimulus characteristics when events are rhythmically expected, as compared with when they are unexpected. From the perspective of dynamic attending theory, quadratic performance profiles of the type observed in the present study are an indication of the degree of synchrony between an internal attentional rhythm and the temporal patterning of stimulus events (Barnes \& M. R. Jones, 2000; M. R. Jones, Moynihan, MacKenzie, \& Puente, 2002; Large \& M. R. Jones, 1999; McAuley \& M. R. Jones, 2003).

The larger auditory tau effect for descending sequences, as compared with ascending sequences, is consistent with research by Henry and McAuley (2009a) on auditory kappa and by Hubbard and colleagues on auditory representational momentum (e.g., Hubbard, 1995). For Hubbard's task, listeners indicated whether the final two tones of an ascending or descending four-tone sequence were the same or different. Memory for the final tone was displaced in the direction of implied motion, as indicated by increased proportions of same responses when the final tone was higher or lower than the penultimate tone, for ascending and descending sequences, respectively. Hubbard reported greater auditory representational momentum for descending sequences than for ascending sequences. Henry and McAuley (2009a) observed a similar effect of direction with respect to the auditory kappa effect; the magnitude of the kappa effect was larger for descending sequences than for ascending sequences for the slow velocity condition. They proposed that if listeners apply an auditory gravity heuristic to tone sequences, descending sequences should be heard as accelerating and, thus, will have faster average velocities than their ascending counterparts. Moreover, if the auditory motion hypothesis operates only within a limited range and the slow velocity sequences are at the slow end of the range, descending sequences may accelerate, so that they are pushed back into the auditory motion range, thus increasing the magnitude of the auditory kappa effect in this condition. Similarly, if, in the present experiment, the descending sequences accelerated in the mind of the listener, the magnitude of the tau effect should be increased, relative to that observed for ascending sequences. More research is needed to better understand the observed direction differences and limits on the range of velocities that produce a sense of auditory motion.

There is some evidence to suggest that individuals with absolute pitch, as well as musically sophisticated listeners, perceive pitch categorically (Burns \& Ward, 1978; Ward, 1982 ) and so may not be expected to show a tau or kappa effect. The present study, as well as Henry and McAuley 
(2009a), did not show any evidence to support this hypothesis. In the two studies, musical training was not correlated with the magnitude of either the tau or the kappa, respectively. However, Shigeno (1993) has provided some additional insight about this issue. Shigeno (1993) demonstrated a tau effect for individuals with absolute pitch in a canonical tau task implementing an AXB paradigm in which, as in the present study, sequences were nonmusical (i.e., the sequences did not establish a strong sense of tonality, and the pitch values of the target tone did not all correspond to musical notes). However, when Shigeno (1993) considered more musical contexts in which bounding tones spanned exactly one octave and all pitch values of the target tone corresponded to musical notes, individuals with absolute pitch did not show an auditory tau effect. Thus, in the context of the present study, it is possible that a musical training effect would have been found if sequences that were more musical had been used.

One potential avenue for future research concerns whether pitch and time cues contribute equally to the effect of velocity on the magnitude of the auditory tau and kappa effects. With respect to kappa, Henry and McAuley (2009a) demonstrated an effect of velocity on the magnitude of kappa by varying the temporal separation between bounding tones, while holding the pitch separation constant. Similarly, MacKenzie (2007) demonstrated the same effect of velocity on the magnitude of kappa by varying the pitch distance between bounding tones, while holding the temporal separation constant. With respect to tau, the present study demonstrated an effect of velocity on the magnitude of the tau by varying the temporal separation between the bounding tones, while holding the pitch separation constant. Previous work by Cohen et al. (1954) and Shigeno (1993) suggests that pitch manipulations of velocity have a similar effect of velocity on the magnitude of the auditory tau effect. Thus, for both auditory kappa and tau, there is converging evidence that both pitch and time cues contribute to the velocity effect; however, the relative contribution of each cue to the magnitude of the velocity effect remains an open question. At least for discrete sequences, there is some support for the view that the temporal separation of the bounding tones will be more effective than the pitch separation in directing listeners' attention to motion-like properties of tone sequences (Henry \& McAuley, 2009b).

\section{Alternative Explanations of the Kappa and Tau Effects}

One potential alternative explanation for the auditory tau and kappa effects that needs to be carefully considered is a response competition hypothesis (Eriksen, 1995; Stroop, 1935). According to this account, tau and kappa effects might arise from simultaneous activation of "competing" responses associated with incongruent pitch-time characteristics of a stimulus. For example, in the context of the canonical auditory tau task, a target tone closer in time to the first tone (A) but closer in pitch to the third tone (B) constitutes an incongruent pairing of stimulus characteristics and, from a response competition perspec- tive, should activate competing responses. Moreover, when incongruent pitch-time pairings are interleaved with trials with congruent pitch-time characteristics (as is the case in the tau task), overall proportions of judgments about the value of the to-be-judged stimulus dimension are predicted to be biased in the direction of the value of the to-be-ignored stimulus dimension (i.e., judgments should show a tau effect).

A response competition account of the tau effect makes several testable predictions. First, response times (RTs) for correct responses to incongruent pitch-time pairs should be lengthened, relative to correct responses to congruent pairs. Second, the probability of making an incorrect response to an incongruent stimulus pairing should increase following a correct response to a congruent stimulus pairing on the preceding trial when the value of the to-be-ignored stimulus dimension on both trials matches. Finally, modifying the proportions of congruent and incongruent trials should affect the magnitude of the auditory tau effect. Specifically, increasing the relative proportion of congruent pitch-time pairings should increase the magnitude of the tau effect, whereas increasing the relative proportion of incongruent pitch-time pairings should decrease the magnitude of the tau effect.

Although testing the latter two hypotheses is beyond the scope of the present study, RT data from Experiment 2 were analyzed to evaluate the first prediction - namely, that RTs for correct responses to congruent trials should be shorter than RTs for correct responses to incongruent trials. For this analysis, a difference score was calculated by subtracting RTs on correct congruent trials from RTs on correct incongruent trials. According to a response competition account, RT difference should be negatively correlated with $w$, and this correlation should be largest at the fastest velocity. Consistent with a response competition account, the overall correlation between RT difference and $w$ was significant $[r(103)=-.39, p<.001]$, but the strength of the relationship did not vary with velocity in the expected manner; when separated by velocity, the only reliable correlation between RT difference and $w$ was found in the medium velocity condition $[r(30)=-.46$, $p<.01]$. Finally, an ANOVA on $w$ scores was rerun using RT difference as a covariate. Notably, for this analysis the effect of velocity on $w$ remained significant $[F(2,98)=$ $\left.7.87, p<.001, \eta_{\mathrm{p}}^{2}=.14\right]$, indicating that RT differences between congruent and incongruent trials at best provide a partial account of the effect of velocity on the magnitude of the tau effect.

Another potential explanation of the tau effect is based on stimulus-response (SR) compatibility. Ishihara, Keller, Rossetti, and Prinz (2008) reported an SR compatibility effect for responses to targets occurring either early or late, relative to a beat instantiated by a preceding periodic click train. Left-side responses were faster and more accurate to early targets, and right-side responses were faster and more accurate to late targets; no compatibility effect was observed when response buttons were aligned vertically. In the present study, the tau effect was characterized by a tendency to make a left-sided response (closer to $A$ ) when 
the timing of the target was early, relative to the temporal bisection point, and to make a right-sided response (closer to $B$ ) when the timing of the target was late, relative to the temporal bisection point. Thus, SR compatibility has the potential to explain aspects of the general tau phenomenon but does not explain the observed effect of velocity on the magnitude of tau. Moreover, SR compatibility for time does not provide a plausible explanation of the kappa effect; however, see Rusconi et al. (2006) for a potential pitch-based SR compatibility approach to the kappa effect.

\section{Broader Implications}

Overall, the results of the present study contribute to a broader theoretical debate in the literature about the relative independence of the processing of pitch- and time-based relations. Strong advocates of a perceptual independence view have argued for both functional and anatomical modularity of pitch and time processing (Peretz, 2006; Peretz \& Coltheart, 2003). Supporting this view are $\mathrm{pitch} /$ time dissociations in individuals with acquired amusia (Ayotte, Peretz, Rousseau, Bard, \& Bojanowski, 2000; Di Pietro, Laganaro, Leemann, \& Schnider, 2004; Griffiths et al., 1997; Johnsrude et al., 2000; Liégeois-Cheval, Peretz, Babai, Laguitton, \& Chauvel, 1998; Tramo, Shah, \& Braida, 2002; Wilson, Pressing, \& Wales, 2002; Zatorre, 1988) and congenital amusia (Foxton, Dean, Gee, Peretz, \& Griffiths, 2004; Hyde \& Peretz, 2004).

There have been increasing challenges to a strict independence view, however. Recently, Foxton, Nandy, and Griffiths (2006) reported that when rhythmic sequences take on dynamic pitch-time trajectories, rhythm perception performance by individuals with congenital amusia deteriorates; normal hearing listeners did not show the same performance decrement when pitch variations were introduced. Converging evidence for the interdependence of pitch and time has been provided for normal-hearing listeners in both nonmusical contexts (Freyd, Kelly, \& DeKay, 1990; Hubbard, 1995; Johnston \& M. R. Jones, 2006) and musical contexts (Ellis \& M. R. Jones, 2009; Lebrun-Guillaud \& Tillmann, 2007).

In nonmusical contexts, in which pitch manipulations do not conform to musical notes or establish a sense of tonality, research on auditory representational momentum reveals that listeners' memory for the final location of a target tone is distorted in the direction of implied motion of an auditory sequence (Freyd et al., 1990; Hubbard, 1995; Johnston \& M. R. Jones, 2006). Moreover, Johnston and Jones (see also Freyd \& Finke, 1984) demonstrated that when irregularities are introduced into the trajectories of auditory patterns, representational momentum is abolished, indicating that forward displacement is dependent on expectations derived from regularities in pitch-time relationships; deviations of pitch-time events from the established trajectory reduce the ability of the perceiver to capitalize on motion-like relations in order to make predictions. Similarly consistent with an auditory motion hypothesis, Hubbard (1995) demonstrated that the magnitude of forward displacement increases with increasing velocity.

In musical contexts, in which sequences establish a sense of tonality and pitch manipulations correspond to musical notes, Lebrun-Guillaud and Tillmann (2007) have demonstrated that listeners' ability to detect deviations from isochrony is influenced by the tonal function of the target tone with respect to the tonality that is established by a preceding chord progression. Temporal change detection was better for the most stable tonic tone than for the unstable leading tone, or the more stable mediant and dominant tones. Taken together, the set of studies described above supports the interactive nature of pitch and time in both nonmusical and musical contexts.

The present study contributes to this debate by adding to support for pitch-time interdependence in nonmusical contexts. Specifically, the demonstration that velocity modulates both auditory tau and kappa (Henry \& McAuley, 2009a) provides converging support for the auditory motion hypothesis. According to this view, processing of pitch- and time-based relations in auditory patterns is fundamentally interdependent within a restricted range of pitch velocities; within this range, attention is guided by stimulus structure according to motion-like properties of stimuli (M. R. Jones, 1976; M. R. Jones \& Yee, 1993; MacKenzie, 2007).

\section{Conclusions}

In conclusion, the results of the present study provide support for an imputed pitch velocity model of the auditory tau effect and the associated auditory motion hypothesis (M. R. Jones, 1976; M. R. Jones \& Yee, 1993; MacKenzie, 2007; MacKenzie \& M. R. Jones, 2005). Combined with research on the auditory kappa effect (Henry \& McAuley, 2009a), the imputed pitch velocity model provides a unified quantitative account of both auditory tau and kappa effects; moreover, with the exception of the visual tau results of Huang and B. Jones (1982), the pattern of findings in the auditory domain is generally consistent with what has been reported for visual kappa and tau (Bill \& Teft, 1972; B. Jones \& Huang, 1982). Converging findings for the effect of velocity on the magnitude of the auditory tau and kappa effects provide further support for an auditory motion hypothesis. In broader terms, this research adds to the growing body of evidence supporting the interdependence of pitch and time relations in auditory pattern perception.

\section{AUTHOR NOTE}

This work was partially supported by NSF Grant BCS-0818271. Portions of this research were presented at the 10th International Conference on Music Perception and Cognition. The authors gratefully acknowledge Gert ten Hoopen, Timothy Hubbard, an anonymous reviewer, and the members of the Rhythm, Attention, and Perception (RAP) Lab at Bowling Green State University for their many helpful suggestions for improvements to earlier versions of the manuscript. Special thanks are also due Bryan Grushcow, Velina Dinkova, Dan Percival, and Nick Steuver for their assistance with data collection. Correspondence concerning this article should be directed to J. D. McAuley, Department of Psychology, Michigan State University, East Lansing, MI 48824 (e-mail: dmcauley@msu.edu).

\section{REFERENCES}

AввE, M. (1936). The spatial effect upon the perception of time. Japanese Journal of Experimental Psychology, 3, 1-52.

ABE, S. (1935). Experimental study on the correlation between time and space. Tohoku Psychologica Folia, 3, 53-68.

Anderson, N. H. (1974). Algebraic models in perception. In E. Car- 
terette \& M. P. Friedman (Eds.), Handbook of perception: Vol. II. Psychophysical judgment and measurement (pp. 258-259). New York: Academic Press.

Ayotte, J., Peretz, I., Rousseau, C., Bard, C., \& Bojanowski, M. (2000). Patterns of music agnosia associated with middle cerebral artery infarcts. Brain, 123, 1926-1938. doi:10.1093/brain/123.9.1926

BARnes, R., \& Jones, M. R. (2000). Expectancy, attention, and time. Cognitive Psychology, 41, 254-311. doi:10.1006/cogp.2000.0738

Benussi, V. (1913). Psychologie der Zeitauffassung. Heidelberg: Carl Winters Universitätsbuchhandlung.

BilL, J. C., \& TeFt, L. W. (1969). Space-time relations: Effects of time on perceived visual extent. Journal of Experimental Psychology, 81, 196-199. doi:10.1037/h0027425

BiLL, J. C., \& Teft, L. W. (1972). Space-time relations: The effects of variations in stimulus and interstimulus interval duration on perceived visual extent. Acta Psychologica, 36, 358-369.

Burns, E. M., \& WARD, W. D. (1978). Categorical perceptionphenomenon or epiphenomenon: Evidence from experiments in the perception of melodic musical intervals. Journal of the Acoustical Society of America, 63, 456-468.

Christensen, I. P., \& Huang, Y. L. (1979). The auditory tau effect and memory for pitch. Perception \& Psychophysics, 26, 489-494.

Cohen, J., Christensen, I., \& ONo, A. (1974). Influence of temporal intervals on comparative judgements of pitch: A study of subjective relativity. Tohoku Psychologica Folia, 33, 76-87.

Cohen, J., Hansel, C. E. M., \& Sylvester, J. D. (1953). A new phenomenon in time judgment. Nature, 172, 901.

Cohen, J., Hansel, C. E. M., \& Sylvester, J. D. (1954). Interdependence of temporal and auditory judgments. Nature, 174, 642-644. doi: $10.1038 / 174642 \mathrm{a} 0$

Cohen, J., Hansel, C. E. M., \& Sylvester, J. D. (1955). Interdependence in judgments of space, time and movement. Acta Psychologica, 11, 360-372.

Collyer, C. E. (1977). Discrimination of spatial and temporal intervals defined by three light flashes: Effects of spacing on temporal judgments and of timing on spatial judgments. Perception \& Psychophysics, 21, 357-364. doi:10.1016/j.humov.2007.07.009

Crowder, R. G., \& Neath, I. (1995). The influence of pitch on time perception in short melodies. Music Perception, 12, 379-386.

Di Pietro, M., Laganaro, M., Leemann, B., \& Schnider, A. (2004). Receptive amusia: Temporal auditory processing deficit in a professional musician following a left temporo-parietal lesion. Neuropsychologia, 42, 868-877. doi:10.1016/j.neuropsychologia.2003.12.004

Douglas, K. M., \& BiLKey, D. K. (2007). Amusia is associated with deficits in spatial processing. Nature Neuroscience, 10, 915-921. doi:10.1038/nn1925

Ellis, R. J., \& Jones, M. R. (2009). The role of accent salience and joint accent structure in meter perception. Journal of Experimental Psychology: Human Perception \& Performance, 35, 264-280. doi:10.1037/a0013482

ERIKSEN, C. E. (1995). The flankers task and response competition: A useful took for investigating a variety of cognitive problems. In C. Bundesen \& H. Shibuya (Eds.), Visual selective attention (pp. 101118). Hillsdale, NJ: Erlbaum.

Foxton, J. M., Dean, J. L., Gee, R., Peretz, I., \& Griffiths, T. D. (2004). Characterization of deficits in pitch perception underlying "tone deafness." Brain, 127, 801-810. doi:10.1093/brain/awh105

Foxton, J. M., NANDY, R. K., \& GRIFFITHS, T. D. (2006). Rhythm deficits in "tone deafness." Brain \& Cognition, 62, 24-29. doi:10.1016/j .bandc.2006.03.005

Freyd, J. J., \& FinKe, R. A. (1984). Representational momentum. Journal of Experimental Psychology: Learning, Memory, \& Cognition, 10, 126-132. doi:10.1037/0278-7393.10.1.126

Freyd, J. J., Kelly, M. H., \& DeKay, M. L. (1990). Representational momentum in memory for pitch. Journal of Experimental Psychology: Learning, Memory, \& Cognition, 16, 1107-1117. doi:10.1037/0278 $-7393.16 .6 .1107$

GeldREICH, E. W. (1934). A lecture-room demonstrator of the visual tau effect. American Journal of Psychology, 46, 483-485. doi: $10.2307 / 1415607$

Gibson, J. J. (1966). The senses considered as perceptual systems. Boston: Houghton Mifflin.
Griffiths, T. D., Rees, A., Witton, C., Cross, P. M., Shakir, R. A., \& GreEN, G. G. R. (1997). Spatial and temporal processing deficits following right hemisphere infarction: A psychophysical study. Brain, 120, 785-794. doi:10.1093/brain/120.5.785

Grondin, S., \& Plourde, M. (2007). Discrimination of time intervals presented in sequences: Spatial effects with multiple auditory sources. Human Movement Science, 26, 702-716. doi:10.1016/j .humov.2007.07.009

HANDEL, S. (1988). Space is to time as vision is to audition: Seductive but misleading. Journal of Experimental Psychology: Human Perception \& Performance, 14, 315-317. doi:10.1037/0096-1523.14.2.315

Helson, H., \& King, S. M. (1931). The tau effect: An example of psychological relativity. Journal of Experimental Psychology, 14, 202-217.

Henry, M. J., \& McAuley, J. D. (2009a). Evaluation of an imputed pitch velocity model of the auditory kappa effect. Journal of Experimental Psychology: Human Perception \& Performance, 35, 551-564.

Henry, M. J., \& McAuley, J. D. (2009b). Relative contribution of frequency and duration cues to estimates of frequency change in tone sequences and glides. Journal of the Acoustical Society of America, 125, 2523

HuANG, Y. L., \& Jones, B. (1982). On the interdependence of temporal and spatial judgments. Perception \& Psychophysics, 32, 7-14.

HubBaRD, T. L. (1995). Auditory representational momentum: Surface form, direction, and velocity effects. American Journal of Psychology, 108, 255-274. doi: $10.2307 / 1423131$

Hyde, K. L., \& Peretz, I. (2004). Brains that are out of tune but in time. Psychological Science, 15, 356-360. doi:10.1111/j.0956 -7976.2004.00683.x

Ishihara, M., Keller, P. E., Rossetti, Y., \& Prinz, W. (2008). Horizontal spatial representations of time: Evidence for the STEARC effect. Cortex, 44, 454-461. doi:10.1016/j.cortex.2007.08.010

Johnsrude, I. S., Penhune, V. B., \& Zatorre, R. J. (2000). Functional specificity in the right human auditory cortex for perceiving pitch direction. Brain, 123, 155-163. doi:10.1093/brain/123.1.155

Johnston, H. M., \& JoNes, M. R. (2006). Higher order pattern structure influences auditory representational momentum. Journal of Experimental Psychology: Human Perception \& Performance, 32, 2-17. doi:10.1037/0096-1523.32.1.2

Jones, B., \& HuANG, Y. L. (1982). Space-time dependencies in psychophysical judgment of extent and duration: Algebraic models of the tau and kappa effects. Psychological Bulletin, 91, 128-142.

Jones, M. R. (1976). Time, our lost dimension: Toward a new theory of perception, attention, and memory. Psychological Review, 83, 323335. doi:10.1037/0033-295X.83.5.323

Jones, M. R., Moynihan, H., MacKenzie, N., \& Puente, J. (2002). Temporal aspects of stimulus-driven attending in dynamic arrays. Psychological Science, 13, 313-319. doi:10.1111/1467-9280.00458

Jones, M. R., \& YeE, W. (1993). Attending to auditory events: The role of temporal organization. In S. McAdams \& E. Bigand (Eds.), Thinking in sound: The cognitive psychology of human audition (pp. 69112). New York: Clarendon Press, Oxford University Press.

Kubovy, M. (1981). Concurrent-pitch segregation and the theory of indispensable attributes. In M. Kubovy \& J. R. Pomerantz (Eds.), Perceptual organization (pp. 58-98). Hillsdale, NJ: Erlbaum.

LARGE, E. W., \& Jones, M. R. (1999). The dynamics of attending: How people track time-varying events. Psychological Review, 106, 119159. doi:10.1037//0033-295X.106.1.119

Lebrun-Guillaud, G., \& Tillmann, B. (2007). Influence of a tone's tonal function on temporal change detection. Perception \& Psychophysics, 69, 1450-1459.

Liégeois-Cheval, C., Peretz, I., Babai, M., Laguitton, V., \& ChauVEL, P. (1998). Contribution of different cortical areas in the temporal lobes to music processing. Brain, 121, 1853-1867. doi:10.1093/ brain/121.10.1853

MacKenZIE, N. (2007). The kappa effect in pitch/time context (Doctoral dissertation, Ohio State University, 2007). Dissertation Abstracts International, 68, 132.

MacKenzie, N., \& Jones, M. R. (2005, November). The auditory kappa effect revisited. Paper presented at the 46th Annual Meeting of the Psychonomic Society, Toronto. 
Macmillan, N. A., \& Creelman, C. D. (1991). Detection theory: A user's guide. New York: Cambridge University Press.

Matsuda, F., \& Matsuda, M. (1979). Effects of spatial separation as a cue of time estimation in children and adults. Japanese Psychological Research, 21, 132-138.

Matsuda, F., \& Matsuda, M. (1981). The anti-kappa effect in successively presented stimuli: A developmental study. Japanese Psychological Research, 23, 9-17.

McAuley, J. D., \& Jones, M. R. (2003). Modeling effects of rhythmic context on perceived duration: A comparison of interval and entrainment approaches to short-interval timing. Journal of Experimental Psychology: Human Perception \& Performance, 29, 1102-1125. doi:10.1037/0096-1523.29.6.1102

Peretz, I. (2006). The nature of music from a biological perspective. Cognition, 100, 1-32. doi:10.1016/j.cognition.2005.11.004

Peretz, I., \& Coltheart, M. (2003). Modularity of music processing. Nature Neuroscience, 6, 688-691. doi:10.1038/nn1083

Price-Williams, D. R. (1954). The kappa effect. Nature, 173, 363-364. doi:10.1038/173363a0

Rusconi, E., Kwan, B., Giordano, B. L., Umiltà, C., \& ButTERWORTH, B. (2006). Spatial representation of pitch height: The SMARC effect. Cognition, 99, 113-129. doi:10.1016/j .cognition.2005.01.004

Sarrazin, J.-C., Giraudo, M.-D., Pailhous, J., \& Bootsma, R. J. (2004). Dynamics of balancing space and time in memory: The tau and kappa effects revisited. Journal of Experimental Psychology: Human Perception \& Performance, 30, 411-430. doi:10.1037/0096 $-1523.30 .3 .411$

Sarrazin, J.-C., Giraudo, M.-D., \& Pittenger, J. B. (2007). Tau and kappa effects in physical space: The case of audition. Psychological Research, 71, 201-218. doi:10.1007/s00426-005-0019-1

SHEPARD, R. N. (1984). Ecological constraints on internal representation: Resonant kinematics of perceiving, imagining, thinking, and dreaming. Psychological Review, 91, 417-447. doi:10.1037/0033 $-295 X .91 .4 .417$

SHigeno, S. (1986). The auditory tau and kappa effects for speech and nonspeech stimuli. Perception \& Psychophysics, 40, 9-19.

SHigENO, S. (1993). The interdependence of pitch and temporal judgments by absolute pitch processors. Perception \& Psychophysics, 54, 682-692.
Stroop, J. R. (1935). Studies of interference in serial verbal reactions. Journal of Experimental Psychology, 18, 643-652.

Tramo, M., Shah, G. D., \& Braida, L. D. (2002). Functional role of auditory cortex in frequency processing and pitch perception. Journal of Neurophysiology, 87, 122-139. doi:10.1152/jn.00104.1999

WARD, W. D. (1982). Absolute pitch. In D. Deutsch (Ed.), The psychology of music (pp. 265-298). New York: Academic Press.

Wilson, S. J., Pressing, J. L., \& Wales, R. J. (2002). Modeling rhythmic function in a musician post-stroke. Neuropsychologia, 40, 1494-1505.

Yoblick, D. A., \& Salvendy, G. (1970). Influence of frequency on the estimation of time for auditory, visual, and tactile modalities: The kappa effect. Journal of Experimental Psychology, 86, 157-164. doi: $10.1037 / \mathrm{h} 0029935$

ZatorRe, R. J. (1988). Pitch perception of complex tones and human temporal-lobe function. Journal of the Acoustical Society of America, 84, 566-572.

\section{NOTES}

1. Because proportions of closer to $B$ responses are plotted on the $y$-axis, the final bounding tone (B) is relatively high for ascending sequences and low for descending sequences. Thus, psychometric curves are flipped for descending sequences, relative to ascending sequences. Proportion of closer to $B$ responses increases for positive target pitch levels for ascending sequences, whereas proportion of closer to $B$ responses decreases for positive target pitch levels for descending sequences.

2. Initially, 130 participants (74 of them female) completed Experiment 2 and were randomly assigned to the fast $(n=47)$, medium $(n=$ $44)$, or slow $(n=39)$ pitch velocity condition. However, 13 participants in the fast velocity condition ( $n=8$, ascending; $n=5$, descending) and 12 in the medium velocity condition $(n=6$, ascending; $n=6$, descending) were dropped from the final sample because of failure to follow instructions or inattention to task. Specific exclusion criteria were (1) negative JNDs (suggesting that participants reversed the instructions) or (2) nonsignificant correlation between target tone pitch level and proportions of closer to $B$ responses. The relative high level of attrition observed is likely due to the fact that a number of participants for this study were recruited from undergraduate classes during the final weeks of the semester, where we have previously observed that the attention level of participants receiving extra credit can be poor. 


\section{APPENDIX}

Imputed Velocity Model

The imputed pitch velocity model was fit to individual participant data from Experiment 2. Predicted proportions of closer to $B$ responses, $P(B)$, were given by

$$
P(B)=f[w p+(1-w) E(p)],
$$

where $p$ was the objective pitch of the target tone in semitones, $E(p)$ was the expected pitch of the target based on the imputed velocity of the sequence, and the parameter $w$ determined the relative contribution of $p$ and $E(p)$. The values for $E(p)$ were derived from imputed velocity, $V_{i}=16 \mathrm{ST} / T$, and the time level, $t$, in milliseconds, of the target tone as follows: $E(p)=V_{i} * t$. The function $f$ was used to map the perceived target pitch, $p^{*}$, where $p^{*}=w p+(1-w) E(p)$, into a closer to $B$ response proportion. Closer to $B$ response proportions were generated for each of the eight target pitch levels $( \pm 75, \pm 150, \pm 225$, and \pm 300 cents) at each of the five target time levels $(-66 \%,-33 \%, 0 \%,+33 \%$, and $+66 \%)$, using

$$
f=\frac{1}{1+e^{-\gamma\left(p^{*}+\theta\right)}}
$$

with two parameters: a gain parameter, $\gamma$, and a bias parameter, $\theta$. In this equation, $p^{*}=w p+(1-w) E(p)$ and denotes perceived target pitch. The value of the bias term, $\theta$, was set to zero for all model fits. The value of the gain, $\gamma$, inversely related to a discrimination threshold, was allowed to vary between participants in order to capture differences in overall discrimination ability, independently of $w$. Model fits were obtained for each participant by minimizing the root-mean squared error of approximation (RMSE_A) between the model's generated response proportions and the actual response proportions, allowing $w$ and $\gamma$ to vary. Overall, the model provided reasonable fits to all the velocity conditions in Experiment 2 (RMSE_A $=0.16$ ), although slightly worse than observed for fits of the model to the auditory kappa effect (RMSE_A $=0.12)$ in Henry and McAuley (2009a). Model fits to the aggregate response proportions (collapsed over participants) provided a better fit (average RMSE_A $=0.06$ ) and the same pattern of results; aggregate $w \mathrm{~s}$ for the fast $(16 \mathrm{ST} / 728 \mathrm{msec})$, medium $(16 \mathrm{ST} / 1,000 \mathrm{msec})$, and slow $(16 \mathrm{ST} / 1,600 \mathrm{msec})$ velocity conditions were $.72, .82$, and .95 , respectively, for ascending sequences and $.76, .77$, and .82 , respectively, for descending sequences. As was expected, the gain, $\gamma$ estimates $( \pm S E M)$ were negatively correlated with JNDs $[r(103)=-.57, p<.001]$. Average values of $\gamma$ for the fast $(16 \mathrm{ST} / 728 \mathrm{msec})$, medium $(16 \mathrm{ST} / 1,000 \mathrm{msec})$, and slow $(16 \mathrm{ST} / 1,600 \mathrm{msec})$ velocity conditions were $0.75( \pm 0.07), 0.92( \pm 0.09)$, and 0.78 ( \pm 0.06$)$, respectively, for ascending sequences and $0.76( \pm 0.08), 0.96( \pm 0.07)$, and $1.11( \pm 0.07)$, respectively, for descending sequences. Aggregate $\gamma$ estimates for the fast $(16 \mathrm{ST} / 728 \mathrm{msec})$, medium $(16 \mathrm{ST} / 1,000 \mathrm{msec})$, and slow $(16 \mathrm{ST} / 1,600 \mathrm{msec})$ velocity conditions were $0.67,0.90$, and 0.69 , respectively, for ascending sequences and $0.70,0.86$, and 0.96 , respectively, for descending sequences.

(Manuscript received July 28, 2008;

revision accepted for publication March 24, 2009.) 